\title{
Apoyo social, sexo y área del conocimiento en el rendimiento académico autopercibido de estudiantes universitarios chilenos
}

\author{
Yaranay López-Angulo² ${ }^{\star}$, María V. Pérez-Villalobos ${ }^{1}$, Rubia C. Cobo-Rendón ${ }^{2}$ y Alejandro E. Díaz-Mujica ${ }^{1}$ \\ (1) Facultad de Ciencias Sociales, Dpto. de Psicología, Universidad de Concepción, Concepción-Chile. \\ (correo-e: marperez@udec.cl; adiazm@udec.cl) \\ (2) Facultad de Ciencias Sociales, Programa de Doctorado en Psicología, Universidad de Concepción, \\ Concepción-Chile (correo-e: yaralopez@udec.cl, rubiacobo@udec.cl)
}

Recibido Jul. 17, 2019; Aceptado Sep. 4, 2019; Versión final Oct. 25, 2019, Publicado Jun. 2020

\section{Resumen}

El objetivo de esta investigación fue analizar las diferencias entre apoyo social percibido, sexo y área del conocimiento sobre el rendimiento académico autopercibido. Participaron 1931 estudiantes de primer y segundo año de universidades chilenas (55.04\% mujeres), con edades entre 17 y 25 años. Se utilizó un cuestionario sociodemográfico y el Cuestionario de Apoyo Social Percibido. Para analizar los datos se realizó un ANOVA factorial inter-sujetos. Se encontró un efecto de interacción estadísticamente significativo del área de conocimiento y apoyo social en el rendimiento académico. La familia predominó como fuente de apoyo social. Existe diferencia por sexo en las variables estudiadas. La percepción de rendimiento académico para las mujeres y hombres fue menor en Ingeniería. Se concluye que el rendimiento académico presenta diferencias en función del apoyo social percibido, el sexo y el área donde pertenece la carrera.

\section{Social support, gender and knowledge area over self-perceived academic performance in Chilean university students}

\begin{abstract}
The objective of this research was to analyze the differences among perceived social support, gender, and knowledge area on self-perceived academic performance. There were 1931 first and second year Chilean university students (55.04\% women) that were surveyed in this study using a socio-demographic questionnaire and the Perceived Social Support Questionnaire. Their ages ranged between 17 and 25 years old. Data was analyzed by applying an inter-subjects factorial ANOVA test. Knowledge area and social support over academic performance showed a statistically significant interaction effect. Families predominated as a source of social support. There was a difference between genders in the variables studied. Academic performance perception for women and men was lower among engineering students. In conclusion, academic performance presents differences with regard to perceived social support, gender, and knowledge area.
\end{abstract}

Keywords: academic performance; social support; university students 


\section{INTRODUCCIÓN}

El rendimiento académico es una variable de relevancia para los estudiantes y la institución educativa. Permite dar cuenta del avance en el cumplimiento de los objetivos educativos y de la asimilación del conocimiento por parte del estudiante (Paba et al., 2008). En la Educación Superior, el rendimiento académico indica que un estudiante se ha adaptado adecuadamente a las exigencias académicas del contexto universitario (Aranda et el., 2013). Desde una perspectiva institucional tiene repercusión en los índices de calidad del sistema educativo (Ezenwoke et al., 2018; Gallegos, et al., 2018); lo cual puede incidir en los procesos de acreditación institucional, asignación de recursos públicos, prestigio y ranking de las universidades.

El rendimiento académico se asocia al grado en que un estudiante logra las metas específicas establecidas por su entorno educativo institucional, relacionado con el nivel de conocimientos mostrados en un área o asignatura particular (Steinmayr et al., 2014). Existe acuerdo en considerar al rendimiento académico como un resultado (calificación o nota) obtenido en las pruebas y exámenes realizados por los estudiantes durante su carrera universitaria (Imose y Barber, 2015; Pérez-Cárceles et al., 2014). Se refiere al nivel de desarrollo que los universitarios alcanzan a través del aprendizaje curricular en un determinado período, con la orientación de los docentes y sobre la base de sus experiencias; considerándose como un proceso de autoevaluación y construcción social de cada estudiante (Tian y Sun, 2018). La percepción del rendimiento académico es lo que se conoce como rendimiento académico autopercibido, relacionado con el modo en que el estudiante percibe y representa su propio desempeño y avance, distinguiéndose de la nota asignada por los profesores. Solo en estudiantes con bajo rendimiento académico se han presentado ligeras diferencias entre el reporte institucional, correspondiente a la nota asignada por el docente y el autorreporte del estudiante (Imose y Barber 2015). El rendimiento académico es una variable determinada por diversos factores que se vinculan a las características personales de cada estudiante, con las puntuaciones alcanzadas en los exámenes en un tiempo específico y las autopercepciones sobre su desempeño y avance.

Los factores relacionados con el rendimiento académico tienen la particularidad de ser específicos y diversos según su naturaleza, algunos estudios han mostrado un listado de factores que predicen el éxito académico en estudiantes universitarios de primer año (Zanden, et al., 2018). Dentro de las variables psicosociales que explican el rendimiento académico es relevante el apoyo social percibido (Richardson et al., 2012). Éste puede definirse como las provisiones instrumentales y/o expresivas, reales o percibidas, que ofrece la comunidad, redes sociales y personas de confianza (Lin et al., 1986); las cuales pueden ser provenientes de tres fuentes: familia, amigos y otras personas significativas (Zimet et al.,1988). El apoyo social percibido se relaciona directamente con el rendimiento académico; los estudiantes que perciben un apoyo social por parte de la familia, amigos y personas en general presentan mayor rendimiento académico (González y Guadalupe, 2017). Se han encontrado diferencias significativas en la percepción de apoyo social en función del sexo, siendo las mujeres quienes presentan mayor percepción de apoyo (Tayfur y Ulupinar, 2016). Las investigaciones no son concluyentes en cuanto a la fuente de apoyo que predice más al rendimiento académico, algunos resultados muestran que el predictor más valioso es la familia (Tayfur y Ulupinar 2016); otros muestran el apoyo de las amistades, seguido del apoyo familiar (Chen et al., 2015) y otros concluyen que el apoyo de los profesores, seguido del apoyo familiar son los más importantes (Fernández-Lasarte et al., 2019). Por tanto, se puede hipotetizar que existen diferencias en los niveles del rendimiento académico autopercibido según las fuentes de apoyo social percibido, teniendo un mayor peso la familia.

Otra variable individual de importancia para el rendimiento académico es el sexo (Pérez-Cárceles et al., 2014; Martínez et al., 2019). Varios estudios han señalado diferencias en el rendimiento académico de los estudiantes universitarios según el sexo; ser mujer ha sido considerado como predictor del alto rendimiento académico en carreras como Agricultura (Khan et al., 2012) en Ciencias de la Salud (Mills et al.,2009) y Enfermería (Wan Chik et al., 2012). Otros estudios refieren que el sexo no es una variable relacionada con el rendimiento académico (Merchán-Galvis et al., 2017; Pérez et al., 2011). Estas investigaciones indican discrepancias entre los hallazgos presentados.

En relación a variables contextuales, existen diferencias en el rendimiento académico según el área del conocimiento en la que se encuentra inserta la carrera que cursa el estudiante (Araque, et al., 2009 y Méndez, 2016). Los estudiantes de carreras pertenecientes a las Ciencias Básicas (Ciencias Biológicas, Ciencias Físicas, o Bioquímica) reportan mayor rendimiento académico que estudiantes de Ingeniería (Porcel et al., 2010). En tal sentido, pareciera que la percepción de rendimiento académico de los estudiantes es diferente según el área del conocimiento.

Comprender el modo en que los factores individuales y sociales relativos a los estudiantes influyen en el rendimiento académico autopercibido entrega elementos para el diseño de políticas públicas relacionadas con la permanencia del estudiante en la universidad y con la calidad de su formación. En la literatura revisada se observan inconsistencias en relación a cómo las fuentes del apoyo social, el sexo y el área del conocimiento 
donde está inserta la carrera marcan diferencias en la percepción de rendimiento académico de los jóvenes universitarios. Por tanto, el objetivo general de esta investigación es analizar las diferencias entre apoyo social percibido, sexo y área del conocimiento sobre el rendimiento académico autopercibido. Para ello, se proponen los siguientes objetivos específicos: 1) Estimar el efecto de interacción entre apoyo social, sexo y área del conocimiento sobre el rendimiento académico autopercibido, 2) Analizar el apoyo social y el sexo respecto al rendimiento académico autopercibido, 3) Analizar el apoyo social y el área del conocimiento respecto al rendimiento académico autopercibido, 4) Analizar el sexo y el área del conocimiento respecto al rendimiento académico autopercibido.

\section{MÉTODO}

El diseño de esta investigación es no experimental de tipo descriptivo transversal, puesto que el estudio se llevó a cabo en el entorno universitario sin ninguna intervención de los investigadores. Es un estudio de tipo transversal debido a que se realizó el proceso de medición en un único momento.

\section{Participantes}

Se realizó un muestreo accidental y según disponibilidad. Participaron 1931 estudiantes (1063 mujeres y 868 hombres) de 7 universidades del sur de Chile, 5 de éstas pertenecientes al Consejo de Rectores de las Universidades Chilenas y 2 privadas. Los estudiantes pertenecían a 70 carreras diferentes y estaban cursando primero y segundo año. Sus edades estuvieron comprendidas entre 17 y 25 años $(M=19.24 ; D E=1.320)$.

\section{Variables e instrumentos de medición}

Apoyo Social Percibido: se midió a través del The Multidimensional Scale of Perceived Social Support (MSPSS), éste cuestionario fue creado para medir percepción de apoyo social proveniente de tres fuentes: familia, amigos y otros significativos y la percepción de apoyo social general, resultante del promedio de las puntuaciones de las tres fuentes (Zimet et al., 1988). Es un instrumento de auto-informe, conformado por 12 ítems con una escala de respuesta tipo Likert que va desde 1= muy en desacuerdo hasta $7=$ muy de acuerdo. Se clasificó como apoyo social bajo cuando el promedio de la puntuación fue menor a 4, medio cuando estaba en el rango de 4 y alto cuando el apoyo percibido estaba entre 5 y 7 . En esta investigación se utilizó la versión en idioma español (Arechabala y Miranda, 2002), que presenta adecuadas propiedades psicométricas, reportando para la dimensión familia y otros significativos un alpha de .86 y para amigos un alpha de .88 .

Sexo, edad, año de ingreso a la carrera, nombre de la universidad y la carrera: se obtuvo a través de un cuestionario sociodemográfico. La variable área del conocimiento se construyó en base a los tipos de carreras y facultades de las universidades participantes, esta distribución fue por elaboración propia. Esta agrupación quedó conformada de la siguiente manera: (1) Ciencias Básicas: Astronomía, Ciencias Físicas, Geofísica, etc. (2) Ingeniería: Industrial, Geomática, Ambiental, Civil, etc. (3) Educación: General, Básica, Diferencial, Parvularia, etc. (4) Salud: Enfermería, Fonoaudiología, Kinesiología, etc. (5) Ciencias Sociales y Humanidades: Comunicación Social, Derecho, Psicología, etc. (6) Arte y Arquitectura: Artes Visuales, Música, Arquitectura, Geografía etc. (7) Administración y Comercio: Auditoría, Negocios Internacionales, e Ingeniería en Administración de Empresas.

Rendimiento académico autopercibido: se solicitó a los participantes reportar la percepción sobre su rendimiento durante el semestre en curso, considerando las puntuaciones de medición para el sistema educativo chileno, siendo una variable continua que va desde $1=$ muy deficiente hasta $7=$ sobresaliente. Se clasificó el rendimiento académico como bajo cuando la calificación fue menor a 3.9, medio cuando estaba en el rango de 4 a 5.9 y alto cuando las calificaciones estaban entre 6 y 7 . El rendimiento académico es el resultado de la percepción del estudiante sobre su desempeño actual.

\section{Procedimiento}

La recogida de datos se realizó en aulas de clases, previa coordinación con las autoridades y con el docente responsable. Se consideraron las normas bioéticas de investigación con seres humanos. El análisis de los datos fue por medio del análisis de la varianza del sexo, área del conocimiento y percepción de apoyo social sobre el rendimiento académico autopercibido, se realizó un ANOVA factorial inter-sujetos. Para ello se corroboró el cumplimiento de supuestos estadísticos de este tipo de prueba. Se asumió el cumplimiento del supuesto de normalidad debido al elevado tamaño de la muestra, siguiendo los postulados de la Teoría del Límite Central. La prueba de Levene, indicó homocedasticidad ( $p>05$ ). Por último, se asumió la independencia de los datos por la construcción del diseño de la investigación. Para el análisis de los datos se utilizó el programa estadístico SPSS 21. 


\section{RESULTADOS}

Para responder al objetivo de estimar el efecto de interacción entre apoyo social percibido, sexo y área del conocimiento sobre el rendimiento académico autopercibido de estudiantes universitarios chilenos, se presenta la tabla 1. Los resultados del ANOVA factorial muestran que para las mujeres existe un efecto estadísticamente significativo del apoyo familiar $\left(F(2,1062)=9.540, p<.001, n^{2} p=.082\right)$, y del apoyo de otros significativos sobre la percepción del rendimiento académico autopercibido $\left(F(2,1062)=17.541, p<.001, n^{2} p=\right.$ .082). Para los hombres existe un efecto estadísticamente significativo del apoyo familiar sobre percepción de rendimiento académico autopercibido $\left(F(2,867)=5.824, p<.005, n^{2} p=.051\right)$ y de apoyo de amigos sobre percepción de rendimiento académico $\left(F(2,867)=3.849, p<.005, n^{2} p=.051\right)$. En el caso del área del conocimiento, los resultados del ANOVA factorial indican que existe un efecto de interacción estadísticamente significativo entre este y el apoyo social percibido respecto al rendimiento académico autopercibido ( $F$ $\left.(12,1930)=2.010, p<.05, n^{2} p=.013\right)$. Es decir, el efecto del apoyo social sobre rendimiento académico autopercibido difiere según el área de estudios donde está inserta la carrera.

Tabla 1: Resultados significativos de efectos inter-sujetos para factores personales y de interacción sobre el rendimiento académico autopercibido.

\begin{tabular}{|l|c|c|c|c|c|c|}
\hline Fuente de apoyo (sexo) & Suma de cuadrados & $g l$ & Media cuadrática & $F$ & Sig. & $n^{2} p$ \\
\hline Apoyo Familiar (mujeres) & 16.618 & 2 & 8.309 & 9.540 & .000 & .082 \\
\hline Apoyo Otro sig. (mujeres) & 30.553 & 2 & 15.277 & 17.541 & .000 & .082 \\
\hline Apoyo Familiar (hombres) & 12.259 & 2 & 6.129 & 5.824 & .003 & .051 \\
\hline Apoyo Amigos (hombres) & 7.698 & 2 & 3.849 & 3.657 & .026 & .051 \\
\hline Área del conocimiento*Apoyo Social General & 24.124 & 12 & 2.010 & 2.159 & .001 & .013 \\
\hline
\end{tabular}

Para analizar el apoyo social y el sexo respecto al rendimiento académico autopercibido se estimaron las diferencias entre estas variables. Los resultados se presentan en la tabla 2. Las comparaciones múltiples, indican que existen diferencias significativas en el rendimiento autopercibido para las mujeres con niveles bajo y alto de apoyo social percibido por parte de la familia y alto en otros significativos $(p<.05)$. En particular, el apoyo social por parte de la familia, presenta diferencias significativas en cuanto al rendimiento académico autopercibido $(p<.05)$, específicamente en el alto apoyo social $(M=4.599, D T=.083)$ respecto a las que perciben bajo apoyo social $(M=4.196, \mathrm{DT}=.094)$. Las mujeres que perciben alto apoyo social por parte de personas significativas tienen mayor rendimiento académico autopercibido que las que tienen apoyo social bajo y medio. También existen diferencias significativas entre las mujeres que perciben alto apoyo social $(\mathrm{M}=$ 4.892, $\mathrm{DT}=.058)$ y las que perciben apoyo social medio $(M=4.083, \mathrm{DT}=.188)$ y bajo $(M=4.248, \mathrm{DT}=.111)$. En conclusión, para el apoyo social percibido por parte de la familia, las diferencias alcanzan en promedio 0.4 puntos, mientras que para el apoyo social por parte de otros significativos alcanzan 0.6 puntos.

Tabla 2: Comparaciones Múltiples según el sexo y nivel de apoyo social.

\begin{tabular}{|c|c|c|c|c|c|c|c|}
\hline $\begin{array}{c}\text { Tipo de apoyo } \\
\text { social }\end{array}$ & \multicolumn{2}{|c|}{ Nivel de apoyo social } & $\begin{array}{c}\text { Diferencias } \\
\text { de medias }\end{array}$ & Desv. Error & Sig & \multicolumn{2}{|c|}{ 95\% de intervalo de confianza } \\
\cline { 5 - 8 } & & & & & Límite inferior & Límite Superior \\
\hline \multirow{2}{*}{ Familia } & Bajo(mujeres) & Alto & -.403 & .094 & .000 & -.628 & -.177 \\
\cline { 2 - 8 } & Bajo (hombre) & Alto & -.418 & .125 & .003 & -.718 & -.119 \\
\hline \multirow{2}{*}{ Otro sig. } & Alto(mujeres) & Bajo & -.645 & .131 & .000 & .331 & -.959 \\
\cline { 2 - 8 } & Alto(mujeres) & Medio & .809 & .198 & .000 & .334 & 1.285 \\
\hline
\end{tabular}

Las comparaciones múltiples, indican que existen diferencias significativas en el rendimiento de los hombres en dependencia del nivel de apoyo social percibido de la familia $(p<.05)$. En particular, hay diferencias significativas, en cuanto al rendimiento académico autopercibido $(p<.05)$, de aquellos que tienen alto apoyo social $(M=4.663, D T=.084)$ respecto a los que perciben bajo apoyo social $(M=4.245$, $D T=.118)$. Esto implica que los hombres que perciben alto apoyo social de la familia presentan mejor rendimiento académico autopercibido que los que perciben apoyo social bajo. En conclusión, al comparar hombres y mujeres, en el caso del apoyo familiar percibido, el efecto es de la misma magnitud (0.4 puntos).

Para analizar el apoyo social y el área del conocimiento respecto al rendimiento académico autopercibido, se estimaron las diferencias entre estas variables, las cuales se presentan en la tabla 3. Las comparaciones múltiples indican que, para los estudiantes de Ingeniería, Educación y Salud, existen diferencias significativas en el rendimiento académico autopercibido de quienes se perciben poco apoyados y los que se perciben muy apoyados $(\mathrm{p}<.05)$. Estas diferencias indican que, a menor apoyo social, menor rendimiento autopercibido por 
el estudiante. En el caso de Ingeniería, hay diferencias significativas en cuanto al rendimiento académico autopercibido $(p<.05)$, de los estudiantes que tienen bajo apoyo social $(M=3.333, D T=.975)$ respecto a quienes tienen medio ( $\mathrm{M}=4.259$, $\mathrm{DT}=1.112)$ y alto $(\mathrm{M}=4.839$, $\mathrm{DT}=1.017)$. Para Educación, hay diferencias significativas en el rendimiento académico autopercibido $(p<.05)$, de quienes tienen bajo apoyo social $(M=4.000, D T=1.247)$ respecto a los que tienen medio $(M=5.014, D T=.954)$ y alto $(M=5.151, D T=.855)$. Para Salud, hay diferencias significativas en cuanto al rendimiento académico autopercibido $(p<.05)$, de quienes tienen bajo apoyo social $(M=3.900, D T=1.100)$ y medio $(M=4.609, D T=1.216)$ respecto a quienes tienen alto $(M=5.120, D T=.858)$. En particular, la diferencia en la percepción de rendimiento de estudiantes con percepción de bajo y alto apoyo es de 1.5 puntos en el área de Ingeniería y de 1.2 puntos en Educación y Salud.

Tabla 3: Comparaciones Múltiples del área del conocimiento y percepción de apoyo social sobre el rendimiento académico autopercibido como variable dependiente.

\begin{tabular}{|c|c|c|c|c|c|c|c|}
\hline \multirow[t]{2}{*}{ Área del conocimiento } & \multirow{2}{*}{\multicolumn{2}{|c|}{ Apoyo Social (General) }} & \multirow{2}{*}{$\begin{array}{l}\text { Diferencia } \\
\text { de } \\
\text { medias }\end{array}$} & \multirow[t]{2}{*}{$\begin{array}{l}\text { Desv. } \\
\text { Error }\end{array}$} & \multirow[t]{2}{*}{ Sig. } & \multicolumn{2}{|c|}{$\begin{array}{c}95 \% \text { de intervalo } \\
\text { de confianza para } \\
\text { diferencia }\end{array}$} \\
\hline & & & & & & $\begin{array}{l}\text { Límite } \\
\text { inferior }\end{array}$ & $\begin{array}{l}\text { Límite } \\
\text { superior }\end{array}$ \\
\hline \multirow[t]{2}{*}{ Ingeniería } & \multirow[t]{2}{*}{ Bajo } & Medio & -.926 & .263 & .001 & -1.556 & -.315 \\
\hline & & Alto & -1.506 & .253 & .000 & -2.113 & -.898 \\
\hline \multirow[t]{2}{*}{ Educación } & \multirow[t]{2}{*}{ Bajo } & Medio & -1.015 & .327 & .006 & -1.798 & -.231 \\
\hline & & Alto & -1.152 & .311 & .001 & -1.897 & -.407 \\
\hline \multirow[t]{2}{*}{ Salud } & Bajo & Alto & -1.220 & .309 & .000 & -1.961 & -.480 \\
\hline & Medio & Alto & -.511 & .130 & .000 & -.838 & -.215 \\
\hline
\end{tabular}

Para analizar el sexo y el área del conocimiento respecto al rendimiento académico autopercibido, se presenta la tabla 4. Existen diferencias significativas en el rendimiento académico autopercibido entre las distintas áreas del conocimiento, las cuales se ven afectadas por el sexo de los estudiantes $(p<.05)$. El rendimiento académico autopercibido de las mujeres es menor en Ciencias Básicas $(M=4.063$, $D T=.175)$ respecto a las mujeres de Educación ( $M=5.115$, $D T=.065)$, de Ciencias Sociales y Humanidades $(M=5.018, D T=.095)$, Arte y Arquitectura $(M=4.955$, $D T=.121)$, Administración y Comercio $(M=5.025, D T=.110)$, Salud $(M=4.991$, $\mathrm{DT}=.054)$ e Ingeniería $(\mathrm{M}=4.746$, $\mathrm{DT}=.070)$. Las estudiantes de Ingeniería presentan menor percepción de rendimiento académico que las de Educación. El rendimiento académico autopercibido de los hombres de Ingeniería ( $M=4.628$, DT=.050) es menor respecto a los hombres de Educación ( $M=5.044$, DT=.093), Salud $(\mathrm{M}=5.113, \mathrm{DT}=.083)$ y Arte y Arquitectura $(\mathrm{M}=5.136, \mathrm{DT}=.122)$. En síntesis, lo común a hombres y mujeres es que existen diferencias en la percepción de rendimiento académico en las carreras que incluyen las áreas de Educación, Salud, Arte y Arquitectura. Las diferencias están en que, para las mujeres, Ciencias Básicas es el grupo de facultades con baja percepción de rendimiento, mientras que para los hombres el área con alta percepción de rendimiento es Arte y Arquitectura.

Tabla 4: Comparaciones Múltiples del sexo y el área de conocimiento sobre la percepción de rendimiento académico.

\begin{tabular}{|c|c|c|c|c|c|c|c|}
\hline \multirow[t]{2}{*}{ Sexo } & \multirow{2}{*}{$\begin{array}{c}\text { Área del } \\
\text { conocimiento }\end{array}$} & \multirow[t]{2}{*}{ Área del conocimiento } & \multirow{2}{*}{$\begin{array}{l}\text { Diferencia } \\
\text { de medias }\end{array}$} & \multirow{2}{*}{$\begin{array}{l}\text { Desv } \\
\text { Error }\end{array}$} & \multirow[t]{2}{*}{ Sig. } & \multicolumn{2}{|c|}{$\begin{array}{l}95 \% \text { de intervalo de } \\
\text { confianza para } \\
\text { diferencia }\end{array}$} \\
\hline & & & & & & $\begin{array}{l}\text { Límite } \\
\text { inferior }\end{array}$ & $\begin{array}{l}\text { Límite } \\
\text { superior }\end{array}$ \\
\hline \multirow{7}{*}{ Mujeres } & \multirow{6}{*}{$\begin{array}{l}\text { Ciencias } \\
\text { básicas }\end{array}$} & Educación & -1.053 & .187 & .000 & -1.622 & -.484 \\
\hline & & Sociales y Humanidades & -1.956 & .199 & .000 & -1.563 & -.349 \\
\hline & & Arte y Arquitectura & -.893 & 213 & .001 & -1.541 & -.244 \\
\hline & & Administración y Comercio & -.962 & 207 & .000 & -1.592 & -.332 \\
\hline & & Salud & -.929 & .183 & .000 & -1.487 & -.371 \\
\hline & & Ingeniería & -.684 & .189 & .006 & .109 & -1.258 \\
\hline & Ingeniería & Educación & -.369 & .095 & .002 & -.659 & -.079 \\
\hline \multirow{3}{*}{ Hombres } & \multirow{3}{*}{ Ingeniería } & Educación & -.416 & .106 & .002 & -1.737 & -.094 \\
\hline & & Salud & -.484 & .097 & .000 & -1.780 & -.189 \\
\hline & & Arte y arquitectura & -.508 & .132 & .003 & -.910 & -.106 \\
\hline
\end{tabular}

\section{DISCUSIÓN}

Los resultados obtenidos en el presente estudio indican que el rendimiento académico autopercibido de los estudiantes es diferente en función del apoyo social, el sexo y área del conocimiento al cual pertenece la carrera. Tanto para hombres como para mujeres, la primera fuente de apoyo es la familia, la cual implica un factor significativo para un mejor rendimiento académico autopercibido. Teniendo en cuenta que el apoyo social puede ser de cuatro tipos (1) emocional, (2) instrumental, (3) evaluativo e (4) informativo (Sarason et. al, 1990); este resultado muestra que la familia es una fuente instrumental y emocional importante en la etapa 
de formación universitaria. Además, es un resultado similar al reportado en otros estudios donde el apoyo social es relevante en la calidad de vida, la salud y el desempeño académico de los estudiantes (Alsubaie et al., 2019; Tayfur y Ulupinar, 2016). De este modo, se refuerza el valor de la familia en esta etapa de la vida, para el desarrollo personal y para el bienestar de los jóvenes.

Al analizar la segunda fuente de apoyo social se identificaron diferencias por sexo; para las mujeres son relevantes otras personas significativas y para los hombres son los amigos. Este resultado coincide con lo presentado por Van der Zanden, et al., (2018), quienes refieren diversos estudios donde el apoyo recibido por los amigos contribuyó positivamente al bienestar durante el primer año. La presencia de apoyo social en la vida de los estudiantes permite, desde la relación con sus amigos, proporcionar consuelo y ánimo en situaciones de estrés, así como tener espacios para compartir experiencias, ideas y opiniones. El apoyo social de la familia proporciona a los estudiantes la posibilidad de expresar las alegrías y frustraciones que los jóvenes experimentan durante su proceso de aprendizaje (Chen et al., 2015).

Se encontraron diferencias entre hombres y mujeres en cuanto a la magnitud del apoyo social, respecto al rendimiento académico autopercibido. Las mujeres que perciben alto apoyo social de la familia y de personas significativas, presentan mayor rendimiento académico que las que perciben apoyo social bajo. En el caso de los hombres, los que perciben alto apoyo social de la familia presentan mayor rendimiento académico que los que perciben apoyo social bajo. Los resultados de este estudio coinciden con otras investigaciones que señalan un mayor rendimiento académico autopercibido en estudiantes que perciben mayor apoyo social por parte de la familia, amigos y personas en general (González y Guadalupe, 2017). La percepción de apoyo social, es relevante para el desarrollo de los estudiantes, tanto en términos académicos como personales, pues influye en la disminución del estrés y la satisfacción de los jóvenes (Novoa y Barra, 2015). Esto implica que los niveles de apoyo social percibido tienen efectos en el proceso de adaptación a la vida universitaria (Zanden, et al., 2018), interviniendo en las formas de responder a las diversas demandas de tipo afectivo y académico presentes en la vida universitaria que afectan la integración social y el rendimiento académico de los estudiantes durante los primeros años de formación (Richardson et al., 2012).

Los resultados obtenidos permiten inferir que el rendimiento académico autopercibido difiere en función del área del conocimiento en la cual se inserta la carrera. Este resultado coincide con el estudio de Araque et. al., (2009) que encuentra diferencias en el rendimiento académico de los estudiantes de diferentes facultades. En nuestros hallazgos los estudiantes de las áreas de Ingeniería y Ciencias Básicas presentaron menor percepción de rendimiento que el resto de las áreas analizadas, esto podría deberse a la presencia de asignaturas críticas en estas carreras donde son elevadas las tasas de reprobación, vinculadas a características institucionales o de cada carrera (Méndez, 2016). El rendimiento académico autopercibido difiere en función del apoyo social percibido, puede constatarse en estudiantes de Ingeniería, Salud y Educación, las cuales indican que, a mayor apoyo percibido, mayor rendimiento académico. Este resultado implica que las facultades deben propiciar que se generen vínculos sociales relevantes que favorezcan el apoyo social, de modo que la alta percepción de apoyo social facilite el incremento del rendimiento académico autopercibido. Este hallazgo es coincidente por lo planteado por Gallegos, et al., (2018), los cuales establecen que una vez que los estudiantes ingresan a la universidad, las intervenciones realizadas desde la carrera permiten a los estudiantes potenciar sus habilidades no solo cognitivas, sino también socioemocionales necesarias para enfrentar con éxito las demandas de la vida universitaria.

Al analizar las particularidades de hombres y mujeres de las diversas áreas del conocimiento respecto al rendimiento académico autopercibido, se encontraron diferencias, siendo las mujeres las que mayores puntuaciones alcanzan; contrariamente a lo propuesto por Merchán-Galvis et al., (2017), donde refieren que el sexo no es una variable relacionada con el rendimiento académico. Se evidenció, que tanto para los hombres como para las mujeres una alta percepción de rendimiento se da para las áreas que incluyen a Educación, Salud, Arte y Arquitectura, mientras que la menor percepción de rendimiento se da para el grupo de estudiantes pertenecientes a Ingeniería. Este hallazgo se vincula con otros estudios que reportan que el tipo de carrera es una variable explicativa del éxito de los estudiantes (Porcel et al., 2010). Las diferencias están en que, para las mujeres, Ciencias Básicas es el grupo con menor percepción de rendimiento, mientras que para los hombres las áreas con mayor percepción de rendimiento son Salud, Arte y Arquitectura. Este resultado difiere de otros estudios donde reportan que las mujeres pertenecientes a carreras de Salud perciben mayor rendimiento académico que sus compañeros (Mills et al., 2009; Wan Chik et al., 2012). Según este resultado las mujeres presentan menor percepción de rendimiento en áreas que son tradicionalmente masculinas, y los hombres tienen mayor percepción en áreas que son tradicionalmente femeninas; dicha percepción pudiera fundamentarse en los estereotipos de género relacionados con los roles de géneros que configuran el modo de percibirse como hombre o mujer ante diferentes tareas.

Los hallazgos de este estudio permiten reforzar la evidencia existente en cuanto a la influencia de diversos aspectos como las características propias del estudiante (sexo, calificaciones anteriores, puntuaciones en 
pruebas de ingreso a la universidad, la carrera elegida), sobre el rendimiento académico (Soria-Barreto y Zúñiga-Jara, 2014) y también otro aspecto como el apoyo social percibido. Aunque en la Educación Superior se realizan acciones para mejorar el rendimiento académico de los estudiantes, queda pendiente incluir y explorar aspectos relativos al apoyo familiar (González y Guadalupe, 2017) y a la generación de vínculos con personas del campus universitario, dada la relevancia que tiene el apoyo social percibido en el rendimiento académico.

La primera limitación de este estudio se relaciona con el alcance en el análisis del apoyo social percibido; si bien, se concluye que son relevantes las fuentes de apoyo social; no se exploró el tipo de apoyo social proporcionados por estas fuentes. La segunda limitación, tiene que ver con el sesgo de deseabilidad social en la medición de la percepción de rendimiento académico, dado que esta puede diferir del rendimiento real obtenido del estudiante en el semestre o curso académico. En este caso la evidencia empírica plantea que, aunque el rendimiento académico percibido tiende a correlacionarse altamente con el rendimiento académico real, existe una ligera pérdida de precisión al momento de ser reportado por los propios estudiantes, en especial cuando los estudiantes presentan bajo rendimiento académico (Imose y Barber, 2015). Por tanto, los resultados obtenidos en este trabajo deben considerar que la evaluación del rendimiento académico se basa únicamente en la percepción del estudiante, lo que hace necesario evaluar el comportamiento de estas variables en relación al rendimiento académico proveniente de las calificaciones obtenidas.

Adicionalmente, puede ser relevante, explorar cómo los estudiantes perciben el apoyo de sus profesores directamente. Algunos estudios han mostrado que el apoyo docente tributa a una mejor adaptación a la universidad y al logro de objetivos académicos (González y Guadalupe, 2017). Es importante, explorar las expectativas de género en cuanto al rendimiento académico en las diversas áreas del conocimiento y otras variables vinculadas con su desarrollo (motivación, autoeficacia). Respecto al área del conocimiento, se hace necesario identificar el tipo de apoyo social que puede proporcionar cada una de estas fuentes (familia, amigos, persona significativa) que influyen en el rendimiento académico y así poder determinar los aspectos que explican las diferencias en la percepción del rendimiento académico encontradas en este estudio.

\section{CONCLUSIONES}

De acuerdo a los resultados y discusión se pueden extraer las siguientes conclusiones principales: 1) El rendimiento académico de los estudiantes es diferente en función del apoyo social percibido, el sexo, y área del conocimiento al cual pertenece la carrera que cursa. 2) Existen diferencias entre hombres y mujeres en cuanto al apoyo social percibido, respecto al rendimiento académico. 3) Existen diferencias entre el apoyo social percibido y el área del conocimiento al cual pertenece el estudiante respecto al rendimiento académico. 4) Existen diferencias entre hombres y mujeres de las diferentes áreas del conocimiento respecto al rendimiento académico.

\section{AGRADECIMIENTOS}

Este trabajo se realizó como parte del Proyecto de investigación más amplio, CONICYT/FONDECYT 1161502, Modelo explicativo de la permanencia y el abandono de los estudios universitarios, basado en procesos cognitivo motivacionales. Cuenta con financiamiento de Becas Doctorado Nacional CONICYT Folio 21170795. Agradecemos a CONICYT por su colaboración.

\section{REFERENCIAS}

Alsubaie, M., Stain, H., Webster, L., y Wadman, R., The Role of Sources of Social Support on Depression and Quality of Life for University Students, https://doi: 10.1080/02673843.2019.1568887, Int J Adolesc Youth, 24(1), 1-13 (2019).

Aranda, A. F., López, V. M., Castejón, F. J., y Romero, R., La Evaluación Formativa en Docencia Universitaria y el Rendimiento Académico del Alumnado, Aula Abierta, ISSN: 0210-2773, 41(2), 23-34 (2013).

Araque, F., Roldán, C., y Salguero, A., Factors Influencing University Drop-out Rates, https://doi:10.1016/j.compedu.2009.03.013, Comput Educ, 53(3), 563-574 (2009).

Arechabala, M. C., y Miranda, C., Validación de una Escala de Apoyo Social Percibido en un Grupo de Adultos Mayores Adscritos a un Programa de Hipertensión de la Región Metropolitana, https://doi: 10.4067/S0717-95532002000100007, Cienc y Enferm, 8(1), 49-55 (2002).

Chen, C.T., Chen, C.F., Hu, J.L., y Wang, C.C., A Study on the Influence of Self-concept, Social Support and Academic Achievement on Occupational Choice Intention, https://doi: 10.1007/s40299-013-0153-2, Asia-Pacific Educ Res, 24(1), 111 (2013).

Ezenwoke, A., Ogunwale, O., y otros cinco autores, Academic Performance Data of Undergraduate Students' in 23 Programmes from a Private University in Nigeria, https://doi: 10.1016/j.dib.2018.07.056, Data Br, 20, 57-73 (2018). 
Fernández-Lasarte, O., Ramos-Díaz, E., Palacios, E. G., y Rodríguez-Fernández, A., Estudio Comparativo entre Educación Superior y Media: Efecto del Apoyo Social Percibido, el Autoconcepto y la Inteligencia Emocional en el Rendimiento Académico, https://doi: 10.5944/educxx1.22526, Educ XX1,1-22 (2019).

Gallegos, J. A., Campos, N. A., Canales, K. A., y González, E.N., Factores Determinantes en la Deserción Universitaria. Caso Facultad de Ciencias Económicas y Administrativas de la Universidad Católica de la Santísima Concepción (Chile) https://doi: 10.4067/S0718-50062018000300011, Form Univ, 11(3), 11-18 (2018).

Chong, E.G, Factores que Inciden en el Rendimiento Académico de los Estudiantes de la Universidad Politécnica del Valle de Toluca, Rev Latinoam Estud Educ, ISSN: 0185-1284, 47(1), 91-108 (2017).

Imose, R., y Barber, L.K., Using Undergraduate Grade Point Average as a Selection Tool: A Synthesis of the Literature, https://doi: 10.1037/mgr0000025, Psychol J, 18(1), 1-11 (2015).

Khan, B., Nawaz, R., y otros tres autores, Evaluation of Comparative Academic Performance of Undergraduate Students at University Level, J Anim Plant Sci, ISSN: 1018-7081, 22(3), 798-801 (2012).

Lin, N., Dean, A., y Ensel, W.M., Social Support, Life Events, and Depression, ed. LTD, 1-371. Academic Press Inc, London, United Kingdom (1986).

Martínez, I.M., Meneghel, I., y Peñalver, J., Does Gender Affect Coping Strategies Leading to Well-being and Improved Academic Performance?, https://doi:10.1016/j.psicod.2019.01.003, Rev Psicodidact, (en prensa) 1-8 (2019).

Méndez, J., Autoconcepto Académico y Rendimiento Académico en Estudiantes de la Universidad de La Frontera. Análisis Comparativo por Facultad, Rev Investig en Educ, ISSN: 0717-6147, 16(1), 169-188 (2016).

Merchán-Galvis, Á.M., Saavedra, H.F., y otros cuatro autores, Estudio de Casos y Controles de Factores Relacionados con el Rendimiento Académico en Estudiantes de Medicina, Educación Médica Superior, ISSN: 1561-2902, 31(3), 101109 (2017).

Mills, C., Heyworth, J., y otros tres autores, Factors Associated with the Academic Success of First Year Health Science Students, https://doi: 10.1007/s10459-008-9103-9, Adv. Health Sci. Educ, 14(2), 205-217 (2009).

Novoa, C., y Barra, E., Influencia del Apoyo Social Percibido y los Factores de Personalidad en la Satisfacción Vital de Estudiantes Universitarios, https://doi: 10.4067/S0718-48082015000300007, Ter Psicol, 33(3), 239-245 (2015).

Paba, C., Lara, R.M., y Palmezano, A.K., Estilos de Aprendizaje y Rendimiento Académico en Estudiantes Universitarios, https://doi: 10.21676/2389783X.661, Duazary, 5(2), 99-106 (2008).

Pérez, C., Ortiz, L., y Parra, P., Prueba de Selección Universitaria, Rendimiento en Enseñanza Media y Variables Cognitivo-actitudinales de Alumnos de Medicina, Rev Educ en ciencias la salud, ISSN: 07-18-2414, 8(2), 120-127 (2011).

Pérez-Cárceles, M. C., Gómez-Gallego, M., y otros tres autores, El Género como Variable Moderadora de los Resultados Académicos en la Enseñanza Universitaria, Estud Econ Reg y Sect , ISSN: 1578-4460, 14(3), 55-64 (2014).

Porcel, E., Dapozo, G., y López, V., Predicción del Rendimiento Académico de Alumnos de Primer Año de la FACENA (UNNE) en Función de su Caracterización Socioeducativa, Rev Electron Investig Educ, ISSN: 1607-4041,12(2), 1-21 (2010).

Richardson, M., Abraham, C., y Bond, R., Psychological Correlates of University Students' Academic Performance: A Systematic Review and Meta-analysis, https://doi: 10.1037/a0026838, Psychol Bull, 138(2), 353-387 (2012).

Sarason, B. R., Sarason, I. G., y Pierce, G. R., Traditional Views of Social Support and their Impact on Assessment. In B. R. Sarason, I. G. Sarason, y G. R. Pierce (Eds.), Wiley series on personality processes. Social support: An interactional view Oxford, England: John Wiley y Sons (1990).

Soria-Barreto, K., Zuniga-Jara, S., y Ruiz-Campo, S., Educación e Intención Emprendedora en Estudiantes Universitarios: un Caso de Estudio, https://doi: 10.4067/S0718-50062016000100004, Form. Univ, 9(1), 25-34 (2016).

Steinmayr, R., Meiner, A., Weidinger, A., y Wirthwein, L., Academic Achievement, Oxford Bibliogtaphies, Oxford, Inglaterra, https://doi: 10.1093/obo/9780199756810-0108 (2014).

Tayfur, C., y Ulupinar, S., The Effect of Perceived Social Support on the Academic Achievement of Health College Students, https://doi: 10.5505/phd.2016.52523, J Psychiatr Nurs, 7(1) 1-6 (2016).

Tian, H., y Sun, Z., Academic Achievement Assessment: Principles and Methodology,1-357, Springer, Berlin, Germany, https://doi: 10.1007/978-3-662-56198-0 (2018).

Van der Zanden, P. J., Denessen, E., Cillessen, A. H., y Meijer, P.C, Domains and Predictors of First-year Student Success: A Systematic Review, https://doi.org/10.1016/j.edurev.2018.01.001, Educational Research Review, 23, 57-77 (2018)

Wan Chik, W., Salamonson, Y., y otros seis autores, Gender Difference in Academic Performance of Nursing Students in a Malaysian University College, https://doi: 10.1111/j.1466-7657.2012.00989, Int Nurs Rev, 59(3), $387-393$ (2012).

Zimet, G. D., Dahlem, N. W., Zimet, S. G., y Farley, G.K., The Multidimensional Scale of Perceived Social Support, https://doi: 10.1207/s15327752jpa5201_2, J Pers Assess, 52(1), 30-41 (1988). 